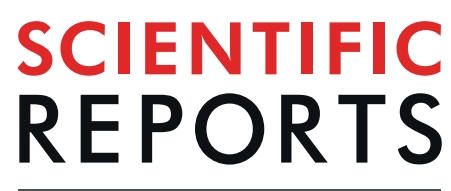

natureresearch

Check for updates

\title{
OPEN Coal-based 3D hierarchical porous carbon aerogels for high performance and super-long life supercapacitors
}

\author{
Yan Lv, Lili Ding, Xueyan Wu, Nannan Guo, Jixi Guo ${ }^{凶}$, Shengchao Hou, Fenglian Tong, \\ Dianzeng Jia ${ }^{\bowtie}$ \& Hongbo Zhang
}

Coal-based 3D hierarchical porous carbon aerogels (3D HPCAs) has been successfully fabricated from a freeze-drying method and with subsequent of calcination process, using coal oxide as carbon precursors, and PVA as both cross-linking agent and sacrifice template. The 3D HPCAs, using as electrode materials for supercapacitors, display outstanding electrochemical performance. The optimal sample (HPCAs-0.4-800) presents a high specific capacitance of $260 \mathrm{Fg}^{-1}$ at $1 \mathrm{Ag}^{-1}$, and exhibits considerable rate capability with the retention of $81 \%$ at $10 \mathrm{Ag}^{-1}$. Notably, HPCAs-0.4-800 shows an excellent cycling stability with $105 \%$ of the capacitance retention after 50000 cycles at $10 \mathrm{~A} \mathrm{~g}^{-1}$, attributing to its unique hierarchical porosity, high surface area up to $1303 \mathrm{~m}^{2} \mathrm{~g}^{-1}$, and improved conductivity. This work offers a promising route to synthesize coal-based porous carbon aerogels electrode materials for supercapacitors.

Supercapacitors (SCs), also called ultracapacitors or electrochemical capacitors, have caused a large amount of interest owing to excellent electrochemical stability, fast charge/discharge, high power density and environmental friendly ${ }^{1-5}$. Supercapacitors store electrical charge on high-surface-area conductive materials, so its performance mainly relies on the electrode materials. Outstanding electrode materials should possess ion approachable high surface areas for high specific capacitance and fasted electron transfer for excellent rate capacity ${ }^{6,7}$. So it is very crucial for supercapacitors with high performance to prepare electrode materials with proper architecture structure, suitable pore size distribution and high specific surface area (SSA) ${ }^{8}$. Among the numerous electrode materials of supercapacitors, carbon materials have attracted more attention because of their unique physical and chemical properties ${ }^{9,10}$. Carbon aerogels (CAs), as one of carbon materials, show outstanding characteristics, such as low density, developed porosity, and multi-branched network structure ${ }^{11-13}$. These structural features can afford the quick transfer channel for ion migration and more active sites, which can lead to the excellent electric double layer performance in supercapacitors. To improve the specific surface area and porosity, most of the CAs are prepared by using pore-forming agents, such as strong bases ${ }^{14-16}$, hard templates ${ }^{17-19}$, soluble salts ${ }^{20,21}$, soft templates and so on ${ }^{22-24}$. Among of them, the soft templates can be directly decomposed during the carbonization process instead of etching procedure using harmful and toxic or corrosive chemicals. Therefore, it has been attracting extensive attention to prepare CAs using soft template for the application of supercapacitors.

Currently, the researches of CAs are mainly focused on precursors, such as resorcinol-formaldehyde ${ }^{25}$, polymers $^{26}$, nanotubes ${ }^{27,28}$, graphene ${ }^{29,30}$, and natural precursors such as cellulose and glucose ${ }^{31,32}$. In our previous works, we have fabricated some functional materials on coal of traditional fossil, such as porous spheres ${ }^{33}$, fibers ${ }^{34}$, bamboo-like carbon nanotubes $(\mathrm{CNTs})^{35}$, graphene quantum dots (GQDs) ${ }^{36}$ and hierarchical porous carbon ${ }^{37}$. All of them demonstrate that the coal can be used to fabricate functional carbon materials. However, so far, coal-based porous carbon aerogels have been few reported and the preparation processes were very complicated and the yield was low in a few studies. Therefore, it is still a great challenge to design simple and productive approaches for the controllable synthesis coal-based porous carbon aerogels.

Key Laboratory of Energy Materials Chemistry, Ministry of Education; Key Laboratory of Advanced Functional Materials, Autonomous Region; Institute of Applied Chemistry, Xinjiang University Urumqi, Urumqi, 830046, P. R.

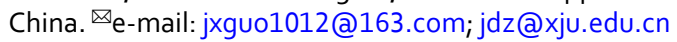




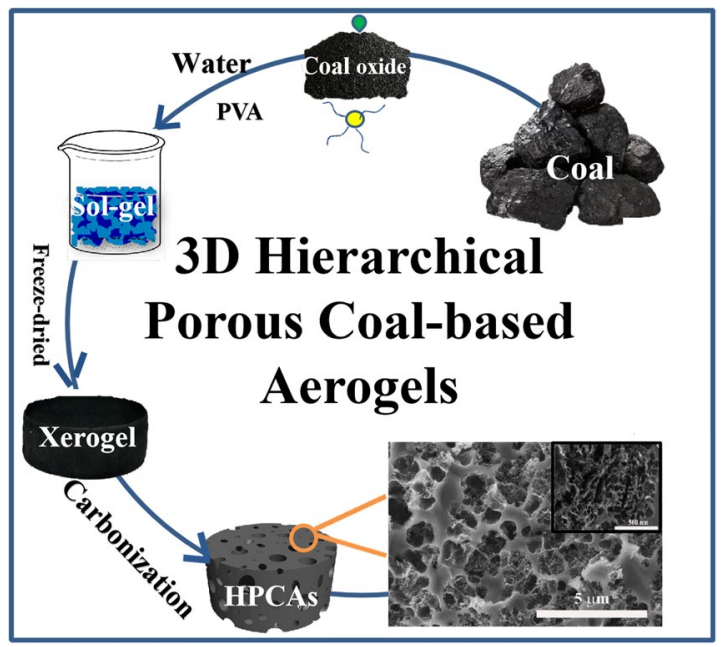

Figure 1. The synthetic scheme of 3D HPCA. (drawn by Microsoft PowerPoint 2010 and 3D Studio Max 8 software).

In our work, we developed an efficient method to construct coal-based 3D HPCAs by carbonization of freezing-dried PVA/coal-based hydrogels, in which coal oxide serves as the carbon source and PVA serves as the sacrificial template and cross-linking agent, respectively. The amount of mesoporous and micropores of the 3D HPCAs can be controlled by tuning the mass ratio of coal oxide and PVA. The performance of the obtained 3D HPCAs are evaluated as the electrode materials of supercapacitors. The optimal sample displays an excellent electrochemical performance. It exhibits a specific capacitance up to $260 \mathrm{Fg}^{-1}$ in the three-electrode system at $1 \mathrm{Ag}^{-1}$, and a high rate performance of $187 \mathrm{Fg}^{-1}$ at $20 \mathrm{Ag}^{-1}$, as well as a remarkable cycling stability ( $105 \%$ of capacitance retention after 50000 cycles). More importantly, the specific capacitance measured was $201.1 \mathrm{~F} \mathrm{~g}^{-1}$ at the current density $1 \mathrm{Ag}^{-1}$ in an assembled symmetrical cell system, and good cycling stability with $108 \%$ over 10000 cycles at $4 \mathrm{~A} \mathrm{~g}^{-1}$. The excellent electrochemical performance may be attributed to the characteristic of 3D cross-linked structure with SSA up to $1303 \mathrm{~m}^{2} \mathrm{~g}^{-1}$, hierarchical porous structure and appropriate ratio of micropore volume to total volume of $65.6 \%$. The materials with hierarchical porous structure can be used as potential electrode materials for energy conversion and storage, and this work provides a green way for high-value utilization of coal in energy storage.

\section{Results and discussion}

The synthesis procedure of the 3D HPCAs is illustrated in Fig. 1. Firstly, PVA/coal-based hydrogels were prepared by using PVA as a crosslinking agent of coal oxide fragment, and then the $3 \mathrm{D}$ network porous structures were formed through a freeze-drying and with subsequent of calcination. To understand the role of PVA and coal oxide in 3D HPCAs, thermogravimetric (TG) analyses of PVA and coal oxide were studied (Fig. S1). The mass loss of PVA is $96.6 \%$ of initial weight from $260^{\circ} \mathrm{C}$ to $490^{\circ} \mathrm{C}$, and $98.8 \%$ when heated to $800{ }^{\circ} \mathrm{C}$, while that of coal oxide is about $62.6 \%$ when heated up to $800^{\circ} \mathrm{C}$ under an argon atmosphere. The results demonstrate that coal oxide is the primary carbon source in the 3D HPCAs, while PVA is the cross-linking agent for formation of hydrogels and the sacrifice template to fabricate $3 \mathrm{D}$ network porous structures.

This result is also directly proved by the optical and SEM images of the pure oxidized coal-800, prepared by the same proceduce only without addition of PVA. As shown in Fig. S2a,b, the pure oxidized coal-800 shows powder shape but non-aerogel on the macro level, and block shape on the micro level, with dense surface and no obvious macropores and mesopores. The morphology of fabricated 3D HPCAs were directly observed by scanning electron microscopy (SEM) and transmission electron microscopy (TEM) (Fig. 2). The SEM images show that the pore structure of 3D HPCAs changed obviously as the increase of PVA content. Consistent well with SEM images, the TEM images of HPCAs-0.4-800 display a richer porous structure and more even pore size distribution obviously. The results demonstrate that an appropriate amount of PVA is crucial for the formation of the pore structure of 3D HPCAs. Coal oxide will stack seriously when crosslinking dose of PVA is excessive, on the contrary, too little sacrificial dose of PVA is not conducive for the formation of holes during the carbonization process. In addition, the influence of calcination temperature on the pore structure of 3D HPCAs was also studied. As shown in Fig. S3, the pore size of HPCAs-0.4-700 and HPCAs-0.4-900 increase due to the change of carbonized temperatures. The broken mesopores can be observed in the HPCAs-0.4-900 due to the skeleton collapse at high carbonization temperature.

The effect of different components and calcination temperatures on the structure of the HPCAs was investigated by X-ray powder diffraction (XRD) (Fig. 3). XRD patterns of 3D HPCAs all display two weak peaks located at $24^{\circ}$ and $43^{\circ}$, associated with diffraction of the (002) and (101) planes carbon. Compare with the peaks of coal oxide (Fig. S4), the peaks around $23^{\circ}$ of the HPCAs shift up to a relatively high angle, indicating that the degree of graphitization is reduced during the process of carbonization ${ }^{39-41}$. In addition, the HPCAs-0.4-800 displays the greatest peak intensity at $23^{\circ}$ among of all HPCAs, indicating that the HPCAs-0.4-800 has the highest degree of graphitization associated with conductivity. Raman spectra are shown in Fig. $3 \mathrm{~b}$ and S3, two obvious peaks 

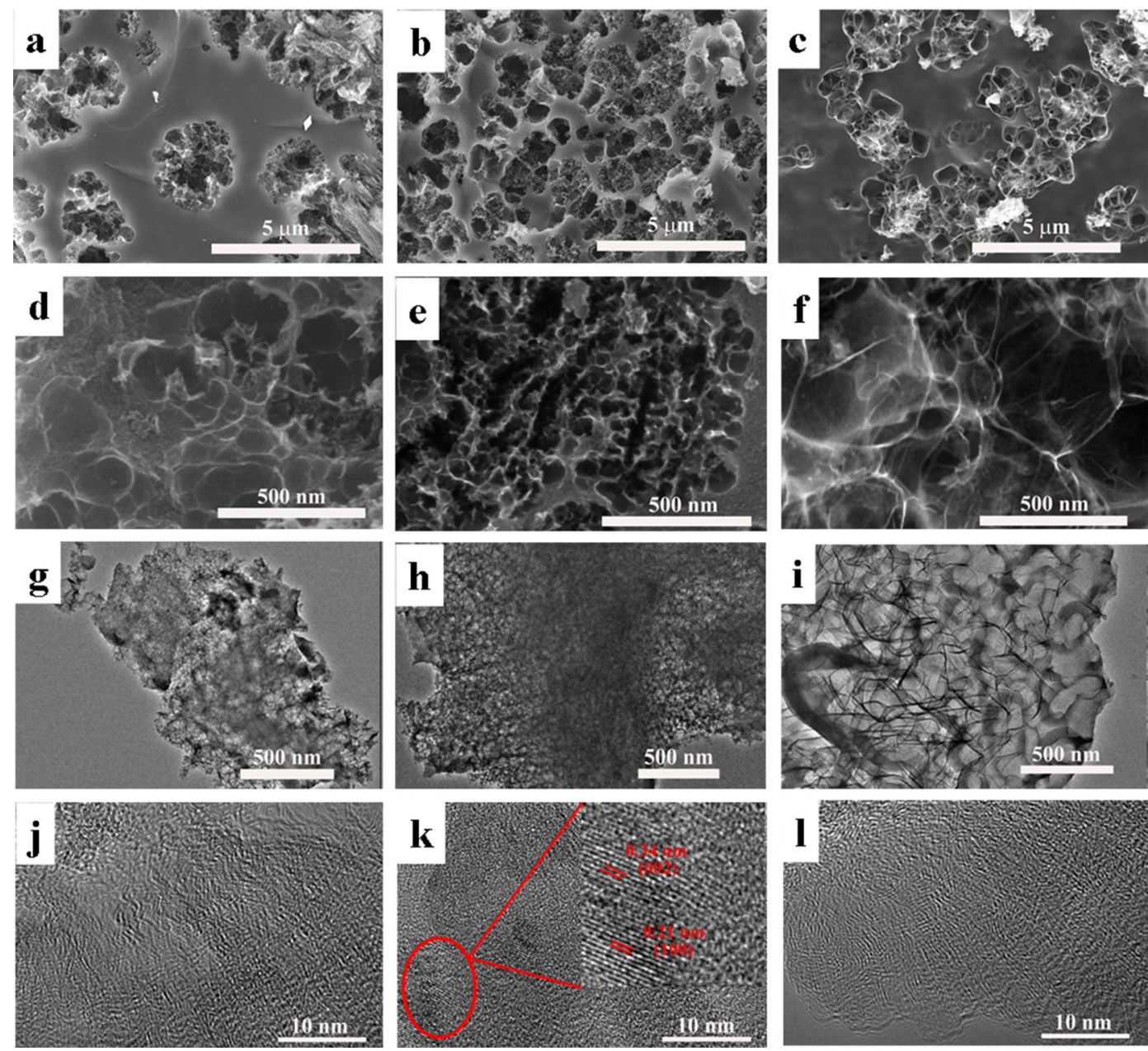

Figure 2. SEM images of 3D HPCAs (a,d) HPCAs-0.2-800; (b,e) HPCAs-0.4-800; (c,f) HPCAs-0.8-800; TEM images of HPCAs (g) HPCAs-0.2-800; (h) HPCAs-0.4-800; (i) HPCAs-0.8-800; HRTEM images of HPCAs (j) HPCAs-0.2-800; (k) HPCAs-0.4-800; (l) HPCAs-0.8-800.
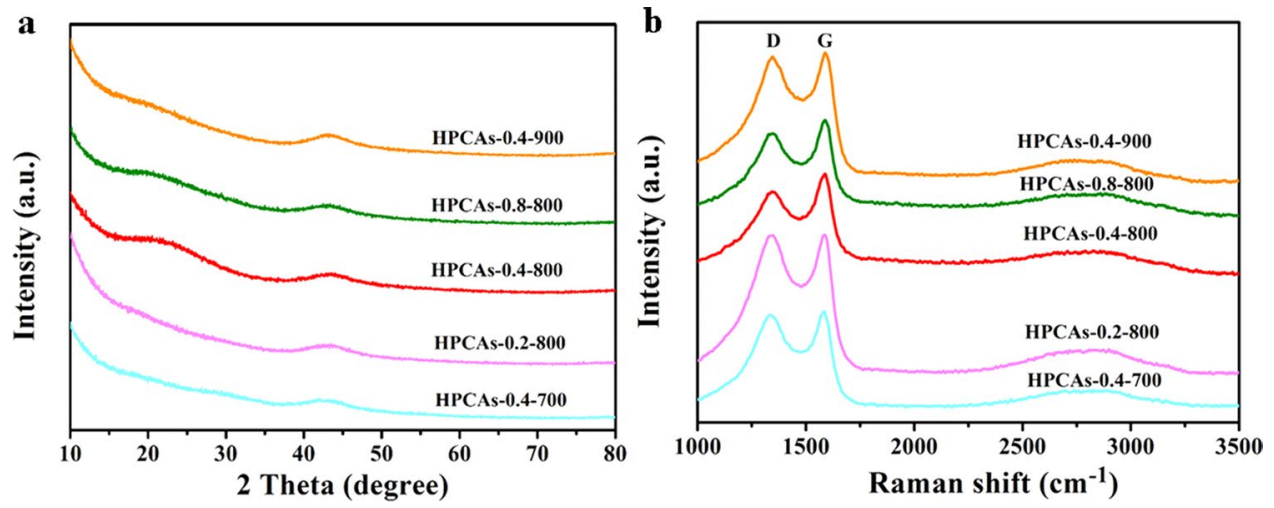

Figure 3. (a) XRD patterns and (b) Raman spectra of 3D HPCAs.

around 1350 and $1590 \mathrm{~cm}^{-1}$ are attributed to D and G bands. The D peak is attributed to the $\mathrm{sp}^{3}$ defects of disordered ones in the hexagonal graphitic layers and $\mathrm{sp}^{2}$ carbon with $\mathrm{O}$-containing groups and $\mathrm{H}$-sites, as well as domain boundary. The $\mathrm{G}$ peak reflects the vibration of $\mathrm{sp}^{2}$-bonded carbon atoms ${ }^{42-44}$. The $\mathrm{I}_{\mathrm{D}} / \mathrm{I}_{\mathrm{G}}$ values of HPCAs0.4-700, HPCAs-0.2-800, HPCAs-0.4-800, HPCAs-0.8-800, HPCAs-0.4-900 and coal oxide are 0.99, 0.97, 0.87, 0.90, 0.97 and 0.81 respectively. Compared HPCAs with coal oxide (Fig. S4), the HPCAs show higher values of $\mathrm{I}_{\mathrm{D}} / \mathrm{I}_{\mathrm{G}}$, because the surface carbon atoms of HPCAs were carried off during the activation operation, thus, leaving the free bond at the surface and forming a disordered carbon structure ${ }^{1,45,46}$. In addition, the HPCAs-0.4-800 has 
the lowest $\mathrm{I}_{\mathrm{D}} / \mathrm{I}_{\mathrm{G}}$ value in the samples of different calcination temperatures, which likely resulted from the reduction reaction at high temperature, on the other hand, the defects of materials increase as the structure collapse at a too high carbonization temperature $\left(900^{\circ} \mathrm{C}\right)$. And among the samples of HPCAs-0.2-800, HPCAs-0.4-800, and HPCAs-0.8-800, HPCAs-0.4-800 also exhibits the highest graphitization degree, which acts as a key role in improving the conductivity. This is because excessive PVA decomposition at high temperatures causes the pore structure to collapse, thus forming an amorphous carbon. The above results prove that appropriate carbonization temperature and PVA content are crucial to the pore structure and the degree of graphitization, which finally reflected in its electrochemical performance.

The XPS spectra were carried out to evaluate the surface atomic composition of HPCAs-0.4-800. Fig. S5 shows the XPS spectra of samples. The survey spectrum confirmed the existence of $\mathrm{C}, \mathrm{O}$, and $\mathrm{N}$ elements in the sample of HPCAs-0.4-800 (Fig. S5a). The results are consistent with the FT-IR spectra data analysis reported in our previous work ${ }^{38}$. The $\mathrm{N}$ and $\mathrm{O}$ in samples are mainly come from the raw coal and nitric acid and sulfuric acid used in the oxidation process. The high-resolution spectrum of C 1s (Fig. S5b) can be divided into three peaks at 288.6, 286.2 and $284.5 \mathrm{eV}$, which are assigned to $\mathrm{C}=\mathrm{O}, \mathrm{C}-\mathrm{O}$ and $\mathrm{C}-\mathrm{C}$, respectively ${ }^{47,48}$. The $\mathrm{O} 1 \mathrm{~s}$ spectrum (Fig. S5c) consists of four peaks located at 530.9, 531.5, 532.4 and $533.5 \mathrm{eV}$, which corresponding to the carbonyl groups $(\mathrm{C}=\mathrm{O})$, bridge-bonded oxygen $(\mathrm{C}-\mathrm{O}-\mathrm{C})$, ester groups $(\mathrm{O}-\mathrm{C}=\mathrm{O})$ and chemisorbed oxygen or water $(\mathrm{COOH}$ carboxylic groups or water), respectively. The high resolution spectrum of $\mathrm{N} 1 \mathrm{~s}$ (Fig. S5d) shows three peaks with binding energy values of $398.1,400.5$ and $404.3 \mathrm{eV}$ for the pyridinic- $\mathrm{N}$, pyrrolic-N and oxidized-N, respectively ${ }^{49,50}$. The elemental contents (atomic\%) of the HPCAs-0.4-800 obtained from XPS data are presented in Table S1. HPCAs0.4-800 has high contents of oxygen and nitrogen, with a ratio of $7.27 \%$ and $1.6 \%$, respectively, which can ameliorate the wettability between the electrode material surface and electrolytes, further facilitate the immersion of electrolyte into the interior of the electrode materials, and ultimately reflect in high capacitance performance. Besides, the pyridinic- $\mathrm{N}$ and pyrrolic- $\mathrm{N}$ can introduce the faradaic pseudocapacitance in aqueous electrolytes and thus also enhance electrochemistry capacitance ${ }^{51}$.

The electric double layer capacitor (EDLC) is a surface regulated phenomenon, so a larger surface area is crucial for acquiring a high capacitance ${ }^{52}$. The $\mathrm{N}_{2}$ adsorption-desorption isotherms and the pore distribution of samples with the different mass ratios of coal oxide/PVA are presented in the Fig. $4 \mathrm{a}-\mathrm{f}$. The isotherms of the 3D HPCAs show classical type-IV curves with an $\mathrm{H} 4$ type hysteresis loop in the relative pressure region between 0.45 and 1.0, suggesting that the existence of a silt-shaped pore structure. Compared with the pure oxidized coal-800 (Fig. S2c), the volume of adsorbed $\mathrm{N}_{2}$ increase steeply at relatively low pressure, which stands for the existence of a large number of micropores, and the remarkable hysteresis loop between $\mathrm{N}_{2}$ adsorption and desorption branch manifests the existence of mesopores. It is well known that micropores and mesopores are in favor of the improvement of charge storage and ion transport, respectively ${ }^{53}$. DFT pore-size-distribution curves show that the HPCAs have broad micropores size distribution $(0.65-2 \mathrm{~nm})$ and a narrow mesopores size distribution $(2-10 \mathrm{~nm})$. The sizes of micropores are close to the size of hydrolyzed $\mathrm{K}^{+}$ions $(0.331 \mathrm{~nm})$, which are beneficial for their capacitive performance ${ }^{54}$. The data of $\mathrm{S}_{\mathrm{BET}}$ and pore distribution of 3D HPCAs are shown in Table 1. $\mathrm{S}_{\mathrm{BET}}$ of HPCAs-0.2-800, HPCAs-0.4-800, HPCAs-0.8-800 are 1018, 1303, and $847 \mathrm{~m}^{2} \mathrm{~g}^{-1}$, respectively. Distinctly, the contribution of micropores to $S_{\mathrm{BET}}$ of HPCAs-0.4-800 (66\%) was greatest among all samples (44\% to HPCAs-0.2-800 and 55\% to HPCAs-0.8-800). In general, the large specific surface area of micropores can result in a high capacity ${ }^{6,55}$. The $\mathrm{N}_{2}$ adsorption-desorption isotherms and the pore distribution of samples with different calcination temperatures (HPCAs-0.4-700 and HPCAs-0.4-900) are shown in Fig. S6. They show similar types of type-IV curves with HPCAs-0.4-800. The effects of the calcination temperature on distribution of hierarchical porous have also been studied, as shown in Table S2. The $\mathrm{S}_{\mathrm{BET}}$ of HPCAs was 971, 1303 , and $900 \mathrm{~m}^{2} \mathrm{~g}^{-1}$ at 700,800 , and $900^{\circ} \mathrm{C}$, respectively. The contribution of micropores to $\mathrm{S}_{\mathrm{BET}}$ of HPCAs0.4-800 (66\%) was also larger than that of HPCAs-0.4-700 (54\%) and HPCAs-0.4-900 (46\%). This is because the high temperature promotes the decomposition of the large amounts of coal oxide and PVA, leading to achieve abundant pores, but too high a temperature $\left(900^{\circ} \mathrm{C}\right)$ will cause the stacking of carbon layers and/or generation of isolated pores, reflecting a smaller $\mathrm{S}_{\mathrm{BET}}$ and the amount of micropores. Such result is related to their electrochemical performances.

The electrochemical performances of 3D HPCAs were evaluated through CV curves at the scan rate of $50 \mathrm{mV} \mathrm{s}^{-1}$ and GCD curves at the current density of $1 \mathrm{Ag}^{-1}$ (Fig. 5a,b). All 3D HPCAs electrode materials display similar rectangles, suggesting that the energy storage type of the 3D HPCAs are the electric double layer capacitor (EDLC). The values of specific capacitance are 232, 260,218, 210 and $184 \mathrm{Fg}^{-1}$ at the current density of $1 \mathrm{Ag}^{-1}$ for HPCAs-0.2-800, HPCAs-0.4-800, HPCAs-0.8-800, HPCAs-0.4-700 and HPCAs-0.4-900, respectively, which is all are superior to that of pure oxidized coal-800 (35.7 $\mathrm{Fg}^{-1}$ Fig. S2e). The 3D HPCAs-0.4-800 exhibits the highest specific capacitance among all the electrode materials due to advantages of the large accessible surface area, more available mesoporous channels and advisable proportion of micropore volume to total volume. As shown in the Fig. 5c,d, specific capacitances of HPCAs-0.4-800 obtained from the discharge curve are 267, 260, 242,224 and $210 \mathrm{Fg}^{-1}$ at the current density of $0.5,1,2,5$ and $10 \mathrm{~A} \mathrm{~g}^{-1}$. On account of the insufficient surface contact and hindrance of ions diffusing into the internal pores, the capacitance decreases as the current density increased $^{56}$. The calculated specific capacitances of the HPCAs at different current densities are presented in Fig. 5e. The 3D HPCAs-0.4-800 electrode material displays the specific capacitance of $260 \mathrm{~F} \mathrm{~g}^{-1}$ at $1 \mathrm{Ag}^{-1}$, which is higher than HPCAs-0.2-800, HPCAs-0.8-800, and the commercial activated carbon (Kuraray YF-50, $87 \mathrm{Fg}^{-1}$ ) (Fig. S7). Such result is probably ascribed to that sufficient micropores and mesopores channel to be used. The specific capacitance of HPCAs-0.4-800 is $210 \mathrm{Fg}^{-1}$ at $10 \mathrm{Ag}^{-1}$, which is about $80.7 \%$ of the capacitance retention of $260 \mathrm{~F} \mathrm{~g}^{-1}$ at $1 \mathrm{Ag}^{-1}$. However, when the current density increases from $1 \mathrm{Ag}^{-1}$ to $10 \mathrm{Ag}^{-1}, \mathrm{HPCAs}-0.2-800$ and HPCAs- $0.8-800$ have the only $74.8 \%$ and $78.9 \%$ capacitance retention, respectively. HPCAs- $0.4-800$ manifests a considerably better rate performance than HPCAs-0.2-800 and HPCAs-0.8-800 samples, because of HPCAs-0.4800 have uniformed and well-interconnected hierarchical porous structure. On the other hands, compared to the 

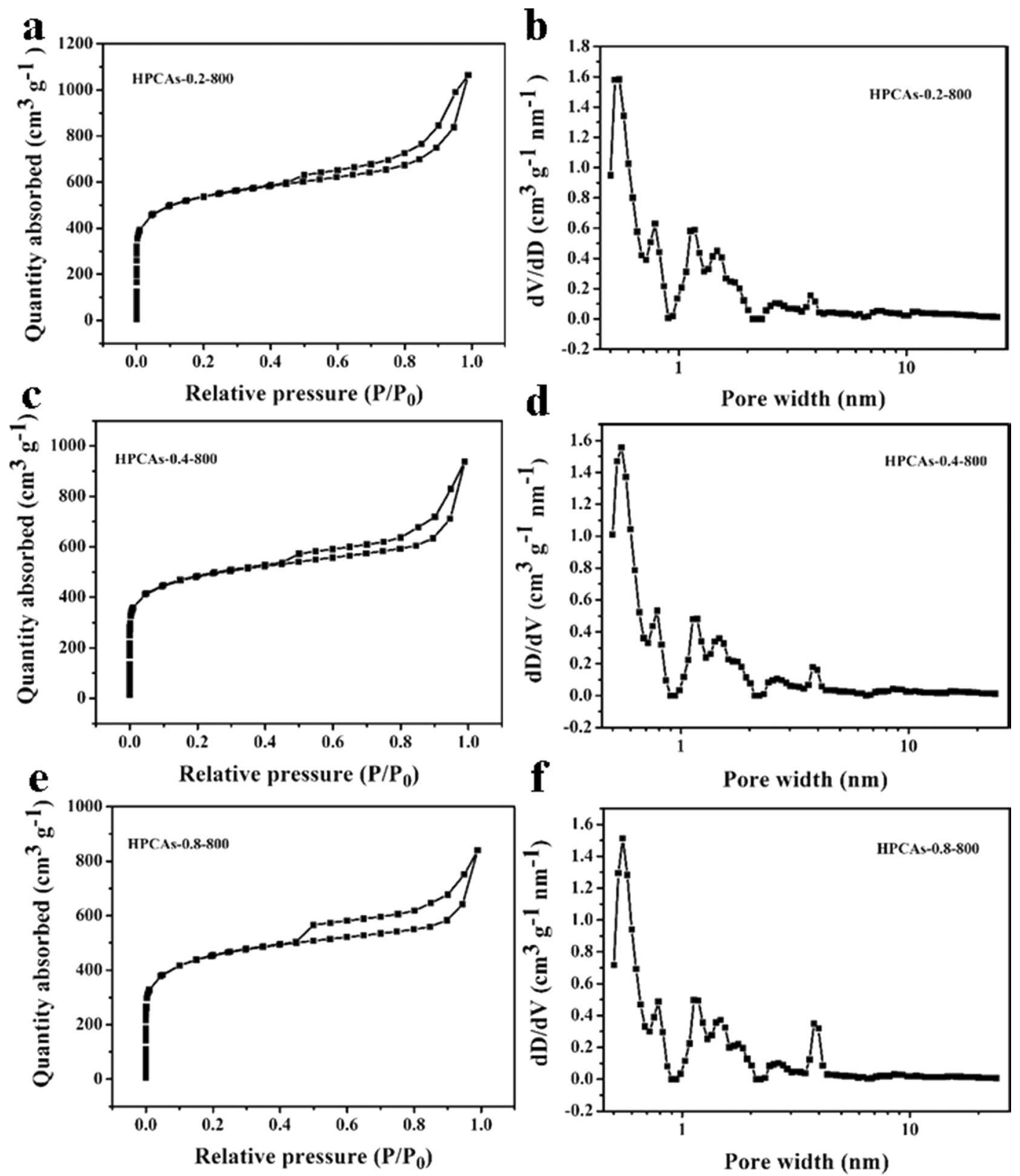

Figure 4. $\mathrm{N}_{2}$ adsorption-desorption isotherms (a,c,e) and pore size distributions (b,d,f) of HPCAs-0.2-800, HPCAs-0.4-800 and HPCAs-0.8-800.

\begin{tabular}{|l|l|l|l|l|l|}
\hline Sample & $\mathbf{S}_{\text {BET }}{ }^{a}\left(\mathbf{m}^{2} \mathbf{g}^{-1}\right)$ & $\mathbf{V}_{\text {total }}{ }^{\mathbf{b}}\left(\mathbf{c m}^{-\mathbf{3}} \mathbf{g}^{-1}\right)$ & $\mathbf{V}_{\text {meso }}{ }^{\mathbf{c}}\left(\mathbf{c m}^{-3} \mathbf{g}^{-1}\right)$ & $\mathbf{S}_{\text {micro }}{ }^{\mathbf{d}}\left(\mathbf{m}^{2} \mathbf{g}^{-\mathbf{1}}\right)$ & $\mathbf{D}_{\text {ap }}{ }^{\mathbf{e}}(\mathbf{n m})$ \\
\hline HPCAs-0.2-800 & 1018 & 1.65 & 0.78 & 450 & 6.4 \\
\hline HPCAs-0.4-800 & 1303 & 1.45 & 0.61 & 856 & 4.4 \\
\hline HPCAs-0.8-800 & 847 & 1.30 & 0.80 & 427 & 6.1 \\
\hline
\end{tabular}

Table 1. BET Specific surface area and porous structure of HPCAs-0.2-800, HPCAs-0.4-800 and HPCAs-0.8800. ${ }^{\mathrm{a}} \mathrm{BET}$ surface area. ${ }^{\mathrm{b}}$ The total pore volume at $P / P_{o}=0.99$, ${ }^{\mathrm{c}}$ The mesopore volume calculated using the $\mathrm{BJH}$ method based on the Kelvin equation. ${ }^{\mathrm{d}}$ Micropore surface area calculated using the V-t plot method. ${ }^{\mathrm{e}}$ Average pore size $\left(4 \mathrm{~V}_{\mathrm{t}} / \mathrm{S}_{\mathrm{BET}}\right)$.

samples of HPCAs-0.4-700 and HPCAs-0.4-900, HPCAs-0.4-800 has the highest SSA and the suitable ratio of micropore volume to total volume of $65.6 \%$, which is good for charge storage. Therefore, it has the highest specific capacitance.

Electrochemical impedance spectroscopy (EIS) were measured to compare the electrochemical kinetics of the samples. Nyquist plots of HPCAs electrode materials consist of a vertical line and a semicircle at the low 

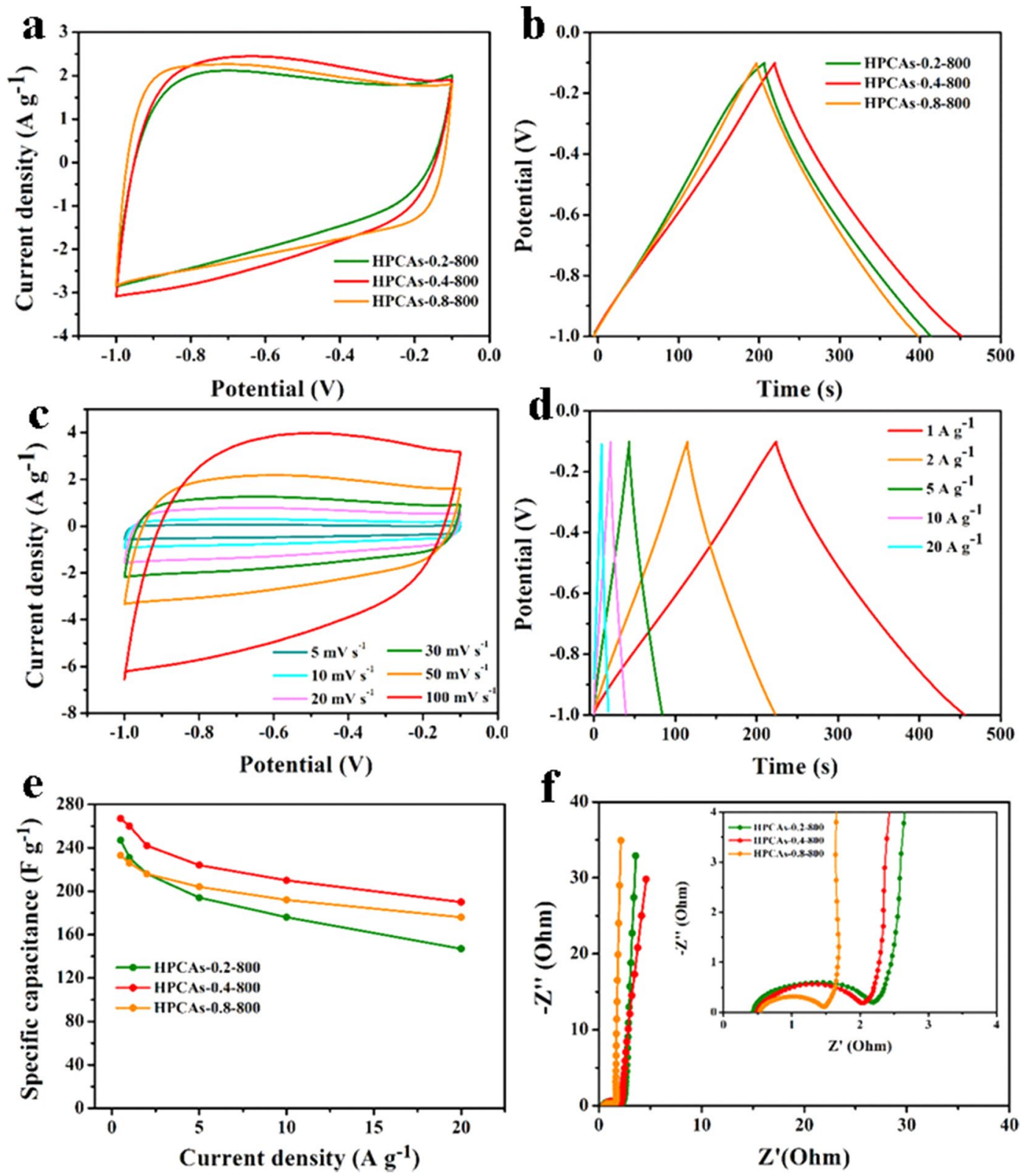

Figure 5. (a) CV curves at the scan rate of $50 \mathrm{mV} \mathrm{s}^{-1}$ and (b) GCD cures at the same scan rate of $1 \mathrm{Ag}^{-1}$ of the samples with different PVA content; (c) CV curves and (d) GCD cures of the HPCAs-0.4-800 sample at different current densities; (e) Specific capacitances of the samples with different PVA content at the current density of $0.5-20 \mathrm{~A} \mathrm{~g}^{-1}$; (f) Electrochemical impedance spectra of 3D HPCAs as Nyquist plots.

frequency and the high frequency area, respectively (Fig. 5f, and S8c). The intercept at the real axis of high frequency of all electrodes are nearly the same, indicating their similar ohmic resistance $\left(\mathrm{R}_{\mathrm{s}}\right)$ of $0.5 \Omega$. A smaller semicircle at the high frequency, reflects a relatively lower charger transfer resistance $\left(R_{c t}\right)^{57}$. The $R_{c t}$ values of HPCAs are 3.1, 2.5, 1.8, 1.5, and 1.1 $\Omega$ for HPCAs-0.4-900, HPCAs-0.4-700, HPCAs-0.2-800, HPCAs-0.4-800 and HPCAs- $0.8-800$, respectively. The HPCAs-0.4-800 has the smallest $\mathrm{R}_{\mathrm{ct}}$ among three samples of different carbonization temperature. The result is due to that HPCAs-0.4-800 has the highest degree of graphitization and comparatively abundant pore structure. Besides, the $\mathrm{R}_{\mathrm{ct}}$ decreased with the increase of the PVA content in samples, which is likely attributed to more available mesoporous channels and more $\mathrm{N}$ content coming from PVA. On the other hand, all samples show nearly perpendicular to the imaginary axis in the low frequency region, which indicated that the electrolyte ions had the best diffusion ability in electrode structure. And the straight line demonstrates the ideal EDLC behavior of electrode materials ${ }^{58,59}$.

From the above results, the HPCAs-0.4-800 exhibits the excellent electrochemical property. The specific capacitance performance of HPCAs-0.4-800 is superior to some previously reported porous carbon materials (Table S3). As mentioned above, the excellent capacitive performance of HPCAs-0.4-800 can be ascribed to the following aspects: (i) the carbon yields of coal oxide and PVA are different, which leads to the formation of 


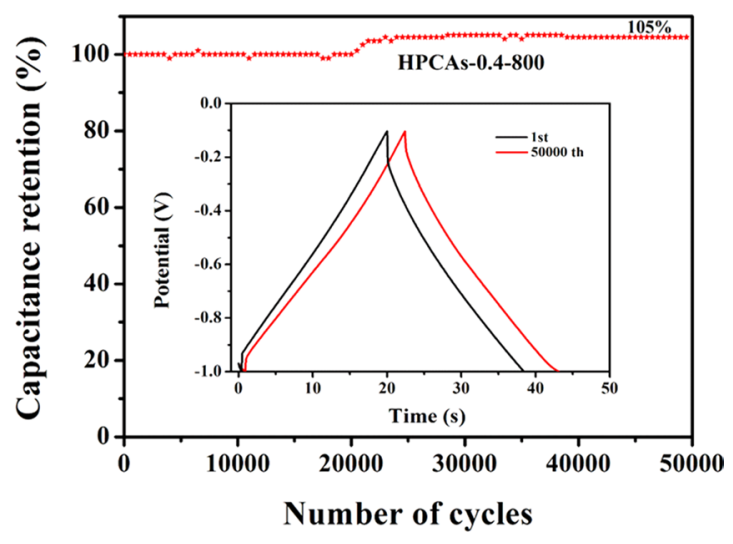

Figure 6. Cycling performance of the HPCAs-0.4-800 electrode at $10 \mathrm{Ag}^{-1}$ (the inset displays GCD cures of the HPCAs-0.4-800 electrode at 1 and after 50000 discharge/charge cycles at $10 \mathrm{Ag}^{-1}$ ).

hierarchical porous materials. (ii) affluent mesopores provide adequate ion transfer passageway. (iii) the unique 3D hierarchical porous structure ensures efficient contact with the electrolyte.

The stable cycling life is an important factor for the practice application of supercapacitor electrode materials. Fig. 6 shows that the specific capacitance of the HPCAs-0.4-800 still reaches $230 \mathrm{~F} \mathrm{~g}^{-1}$ at the high density of $10 \mathrm{~A} \mathrm{~g}^{-1}$, and superior capacitance is maintained up to $105 \%$ after 50000 cycles. What impressed us most is that the capacitance presents an increasing trend during the repeating process of cycling at the high current density of $10 \mathrm{~A} \mathrm{~g}^{-1}$. On the basis of the pore distribution, this phenomenon has to do with the porous structure of the electrode materials. To be specific, in the beginning stages, only large pores and mesopores are infiltrated by electrolyte, the micropore structure is not fully utilized due to the thin film on the electrode of supercapacitors ${ }^{60}$. However, the $\mathrm{K}^{+}$hydrated ions can gradually penetrate into the micropores and participate in the establishment of electric double layers ${ }^{61}$. The electrode material of 3D HPCAs-0.4-800 tends to a stable capacitance value due to the full utilization of pores as the cycles increasing. Therefore, the sample has a considerable reversibility and satisfactory cycle stability during the repeated charge-discharge process.

Simultaneously, the electrochemical performance of the HPCAs-0.4-800 in symmetric cell system was also investigated. Fig. 7a shows that the HPCAs-0.4-800 has rectangular CV curves at different scan rates, indicating distinct capacitive behavior and fine reversibility. Fig. 7b shows the galvanostatic charge-discharge (GCD) curves at different current densities from 1 to $20 \mathrm{Ag}^{-1}$ in the potential range from 0 to $1 \mathrm{~V}$. The HPCAs-0.4-800 shows an excellent specific capacitance of $201.1 \mathrm{Fg}^{-1}$ at $1 \mathrm{Ag}^{-1}$ and $160.0 \mathrm{~F} \mathrm{~g}^{-1}$ at $20 \mathrm{Ag}^{-1}$, respectively. And the electrode has a good rate capability with about $80 \%$ capactive retention at $20 \mathrm{Ag}^{-1}$. As shown in Fig. $7 \mathrm{c}$, the HPCAs-0.4800 -based device has a pretty high energy density $\left(7.2 \mathrm{Wh} \mathrm{kg}^{-1}\right.$ at $\left.500 \mathrm{~W} \mathrm{~kg}^{-1}\right)$. Fig. $7 \mathrm{~d}$ manifests the cycling stability of the HPCAs-0.4-800//HPCAs-0.4-800 cell. The specific capacitance retention up to $108 \%$ after 10,000 cycles due to the fully wetting of sufficient pores during the long time charged/discharged process, exhibiting its outstanding stable cycling. To sum up, the excellent electrochemical performances of HPCAs-0.4-800, such as high specific capacitance, and good cycling stability can be attributed to the $3 \mathrm{D}$ hierarchical porous and the appropriate microspores and mesopores size distribution as well as improved conductivity.

\section{Conclusion}

In summary, we design a low cost and facile strategy to obtain coal-based 3D HPCAs by carbonization of the freeze dried PVA/coal-based hydrogels. The structure and electrical performances of 3D HPCAs are adjusted and optimized by changing the content of PVA and carbonization temperature. Among of all samples, the HPCAs-0.4-800, as an electrode material of supercapacitors, exhibits excellent specific capacitances of $260 \mathrm{Fg}^{-1}$ and $201.1 \mathrm{~F} \mathrm{~g}^{-1}$ at $1 \mathrm{Ag}^{-1}$ in $6.0 \mathrm{M} \mathrm{KOH}$ electrolyte for the three- and two-electrode systems, respectively. It also displays an excellent cycling stability of $105 \%$ capacitance retention after 50000 cycles. This work provides a promising route to construct coal-based 3D HPCAs as highly efficient electrodes materials for supercapacitors.

\section{Experiment Section}

Materials. Coal was obtained from Heishan, Xinjiang, China. The similar analysis of coal has been reported by our group ${ }^{38}$. Polyvinyl alcohol (PVA) (molecular weight is $44.05 \mathrm{MW}$ ) purchased from Sigma-Aldrich. $\mathrm{H}_{2} \mathrm{SO}_{4}$ (98\%), $\mathrm{HNO}_{3}(63 \%), \mathrm{KOH}$ were analytical grade.

The preparation of 3D HPCA. Coal oxide was firstly obtained by treating raw coal using a mixed acid $\left(\mathrm{VHNO}_{3} / \mathrm{VH}_{2} \mathrm{SO}_{4}=1: 3\right)$ as previously reported by our group ${ }^{38}$. Then PVA/coal-based hydrogels were synthesized as following processes. Firstly, coal oxide $(0.8 \mathrm{~g})$, PVA $(0.4 \mathrm{~g})$ were dissolved in $10.0 \mathrm{~mL}$ deionized water and the $\mathrm{pH}$ of the solution was neutralized with the $\mathrm{KOH}$, and then stirred at $80^{\circ} \mathrm{C}$ continuously for $12 \mathrm{~h}$. Secondly, the product was frozen in refrigerator $\left(-70^{\circ} \mathrm{C}\right)$ for $24 \mathrm{~h}$ and freeze-dried in vacuum for $24 \mathrm{~h}$ to obtain the xerogels. Finally, the as prepared xerogel was calcined at $800^{\circ} \mathrm{C}$ for $2 \mathrm{~h}$ with a heating rate of $5^{\circ} \mathrm{C} \mathrm{min}-1$ under flowing $\mathrm{N}_{2}$ atmosphere for formation of 3D coal-based HPCAs, the sample was called HPCAs-0.4-800. Similarly, HPCAs0.2-800 and HPCAs-0.8-800 were prepared by the nearly same methods with the only different mass ratios of coal 

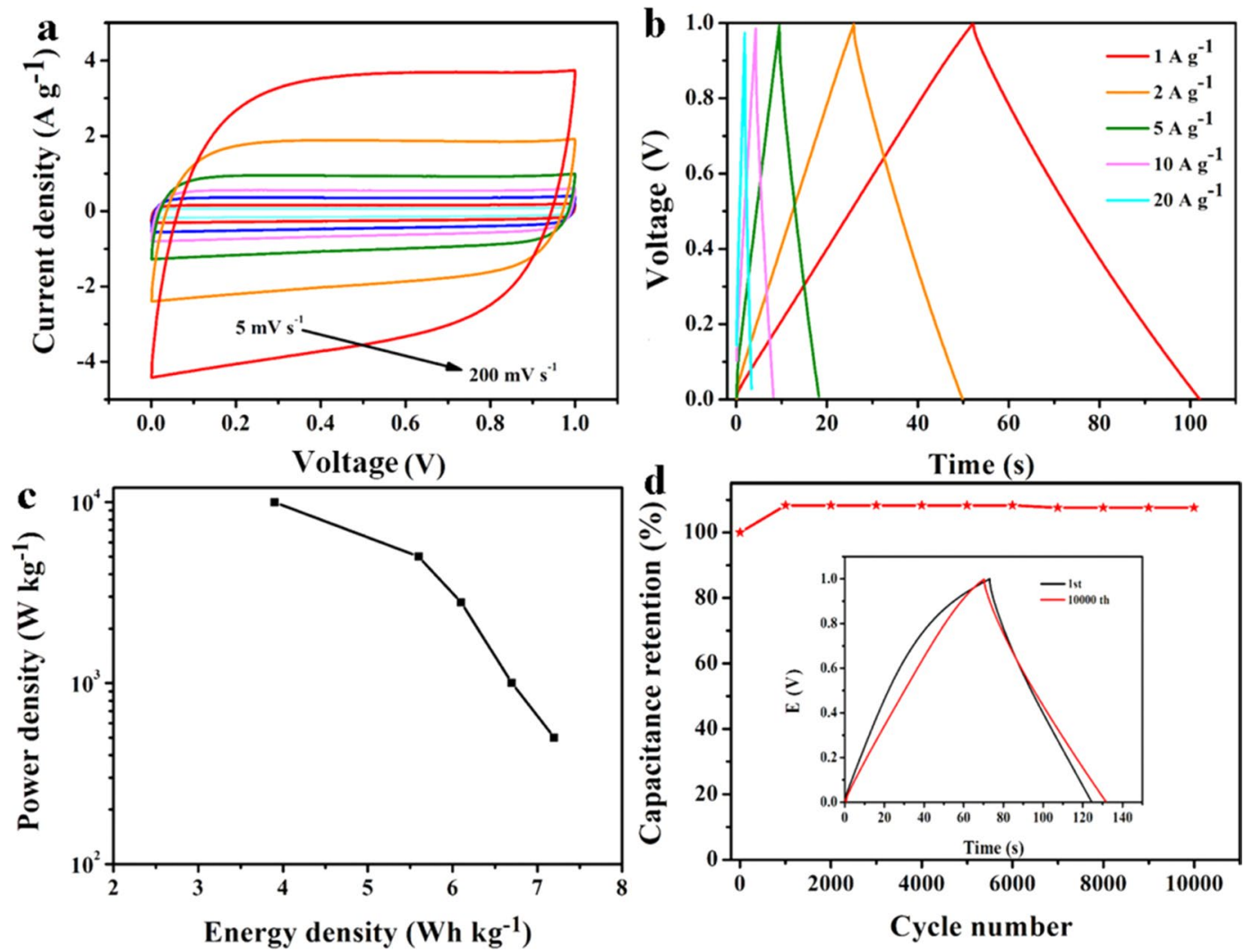

Figure 7. Electrochemical performances of the HPCAs-0.4-800//HPCAs-0.4-800 symmetric cell: (a) CV curves at various sweep rates. (b) GCD curves recorded at 1-20 $\mathrm{Ag}^{-1}$. (c) Ragone plot. (d) Cycling stability measured at $4 \mathrm{Ag}^{-1}$ with inset showing GCD curves $\left(1 \mathrm{Ag}^{-1}\right)$ before and after 10,000 cycles.

oxide/PVA $(0.8 / 0.2,0.8 / 0.8)$. For the two others of coal based HPCAs annealed at $700^{\circ} \mathrm{C}$ (denoted as HPCAs-0.4700 ) and $900^{\circ} \mathrm{C}$ (denoted as HPCAs- $0.8-900$ ) were synthesized for comparison. And the pure oxidized coal-800, as a control sample, was prepared by the same methods only without addition of PVA and annealed at $800^{\circ} \mathrm{C}$.

Structural characterization. SEM and TEM images were recorded on field emission scanning electron microscopy (FESEM Hitachi SU-4800) and transmission electron microscopy (TEM, JEM-2100F), respectively. Thermogravimetric analysis (TGA) was tested by using a NETZSCH STA449F3-QMS403C instrument under $\mathrm{N}_{2}$. XRD measurements were carried out on an X-ray diffractometer (XRD, Bruker D8, using filtered $\mathrm{Cu} K \alpha$ radiation). X-ray photoelectron spectroscopy (XPS) spectra and the Raman spectrum were recorded with a Thermo ESCALAB 250 instrument (Al Ka X-ray source) and a Bruker Senterra spectrometer ( $532 \mathrm{~nm}$ ) Raman spectrometer, respectively. SSA and pore size distribution of coal-based 3D HPCAs were determined on Autosorb-IQ, Quantachrome by BET method.

Electrochemical characterization. The electrochemical experiments of coal-based 3D HPCAs were tested in the three-electrode system in $6.0 \mathrm{M} \mathrm{KOH}$ electrolyte at room temperature, in which the 3D HPCAs use as working electrode, $\mathrm{Ag} / \mathrm{AgCl}$ and $\mathrm{Pt}$ foil $\left(1 \mathrm{~cm}^{2}\right)$ as the reference electrode and counter electrode, respectively. The working electrodes were prepared by mixing 3D HPCAs, carbon black and [Poly (trafluoroethylene)] at a mass ratio of 8:1:1 in absolute ethyl alcohol (the active mass is about $2.0 \mathrm{mg}$ ), and then the mixture was pasted on the nickel form, and dried at $60^{\circ} \mathrm{C}$ for $12 \mathrm{~h}$. The electrochemical performances of these electrodes were carried out using the CHI 660D electrochemical workstation (Chenhua, China). The current density of $10 \mathrm{Ag}^{-1}$ was applied in cyclic GCD measurements for over 50,000 cycles (the potential is $-1.0--0.1 \mathrm{~V}$ ). The specific capacitance $\left(\mathrm{C}_{\mathrm{S}}\right)$ of the $3 \mathrm{D}$ HPCAs electrode materials were calculated via Eq. (1).

$$
\mathrm{C}_{\mathrm{S}}=\mathrm{I} \times \Delta \mathrm{t} /(\mathrm{m} \times \Delta \mathrm{V})
$$

Where I, $\Delta \mathrm{t}, \Delta \mathrm{V}, \mathrm{m}$ is current, discharging time, potential window and the mass of the active material, respectively.

In two-electrode system, $\mathrm{CV}$ and GCD curves were measured using the product in $6.0 \mathrm{M} \mathrm{KOH}$ as the electrode (the total mass of the active on two working electrodes is $4.0 \mathrm{mg}$ ). The specific capacitance for the single electrode $\left(C_{\text {sp }}\right)$ was obtained via Eq. (2). 


$$
\mathrm{C}_{\mathrm{sp}}=4 \mathrm{I} \times \Delta \mathrm{t} /(\mathrm{M} \times \Delta \mathrm{V})
$$

Where, $\Delta \mathrm{V}$ refers to the voltage range, $\mathrm{M}$ represents the total mass of the active materials on two working electrodes, $\Delta \mathrm{t}$ is the discharge time, $\mathrm{I}$ is the discharge current.

$$
\begin{gathered}
\mathrm{E}=\mathrm{C}_{\mathrm{sp}} \Delta \mathrm{V}^{2} /(8 \times 3.6) \\
\mathrm{P}=3600 \times \mathrm{E} / \Delta \mathrm{t}
\end{gathered}
$$

Where E, $\mathrm{P}$ are the energy density $\left(\mathrm{Wh} \mathrm{kg}^{-1}\right)$, power density $\left(\mathrm{W} \mathrm{kg}^{-1}\right)$, respectively.

Received: 9 January 2020; Accepted: 9 April 2020;

Published online: 27 April 2020

\section{References}

1. Zhao, H. et al. Coal based activated carbon nanofibers prepared by electrospinning. J. Mater. Chem. A 2, 9338-9344 (2014).

2. Liu, C., Li, F., Ma, L. \& Cheng, H. Advanced materials for energy storage. Chem. Mater. 24, 1192-1197 (2012).

3. Li, Y. et al. Ni-Co sulfide nanowires on nickel foam with ultrahigh capacitance for asymmetric supercapacitors. J. Mater. Chem. A 2, 6540-6548 (2014).

4. Fan, W. et al. Graphene wrapped polyaniline hollow spheres as novel hybrid electrode materials for supercapacitor applications. ACS Appl. Mater. Interf. 5, 3382-3391 (2013).

5. Miao, Y., Fan, W., Chen, D. \& Liu, T. High-performance supercapacitors based on hollow polyaniline nanofibers by electrospinning. ACS Appl. Mater. Interf. 5, 4423-4428 (2013).

6. Zhai, T. et al. Phosphate ion functionalized $\mathrm{Co}_{3} \mathrm{O}_{4}$ ultrathin nanosheets with greatly improved surface reactivity for high performance pseudocapacitors. Adv. Mater. 29, 1604167-1604174 (2017).

7. Yang, T. et al. From biomass with irregular structures to $1 \mathrm{D}$ carbon nanobelts: a stripping and cutting strategy to fabricate high performance supercapacitor materials. J. Mater. Chem. A 5, 14551-14561 (2017).

8. Duan, B. et al. Unique elastic N-doped carbon nanofibrous microspheres with hierarchical porosity derived from renewable chitin for high rate supercapacitors. Nano Energy 27, 482-491 (2016).

9. Inagaki, M., Konno, H. \& Tanaike, O. Carbon materials for electrochemical capacitors. J. Power Sources 195, 7880-7903 (2010).

10. Pachfule, P., Shinde, D., Majumder, M. \& Xu, Q. Fabrication of carbon nanorods and graphene nanoribbons from a metal-organic framework. Nat. Chem. 8, 718-724 (2016).

11. Li, G. et al. Mesoporous $\mathrm{MnO}_{2}$ /carbon aerogel composites as promising electrode materials for high-performance supercapacitors. Langmuir. 26, 2209-2213 (2010).

12. Hamedi, M. et al. Nanocellulose aerogels functionalized by rapid layer-by-layer assembly for high charge storage and beyond. Angew. Chem. Int. Ed. 52, 12038-12042 (2013).

13. Yang, H., Zhang, T., Jiang, M., Duan, Y. \& Zhang, J. Ambient pressure dried graphene aerogels with superelasticity and multifunctionality. J. Mater. Chem. A 3, 19268-19272 (2015).

14. Han, J. et al. Facile green synthesis of 3D porous glucose-based carbon aerogels for high-performance supercapacitors. Electrochim. Acta. 258, 951-958 (2017).

15. Jiang, J. et al. Sol-gel process-derived rich nitrogen-doped porous carbon through $\mathrm{KOH}$ activation for supercapacitors. Electrochim. Acta. 158, 229-236 (2015).

16. Zhu, Y. et al. Carbon-based supercapacitors produced by activation of graphene. Science 332, 1537-1541 (2011).

17. Ma, F. et al. Construction of 3D nanostructure hierarchical porous graphitic carbons by charge-induced self-assembly and nanocrystal-assisted catalytic graphitization for supercapacitors. Chem. Commun. 52, 6673-6676 (2016).

18. He, X. et al. N-Doped porous graphitic carbon with multi-flaky shell hollow structure prepared using a green and 'useful' template of $\mathrm{CaCO}_{3}$ for VOC fast adsorption and small peptide enrichment. Chem. Commun. 53, 3442-3445 (2017).

19. Chang, B., Zhang, S., Sun, L., Yin, H. \& Yang, B. 2D graphene-like hierarchically porous carbon nanosheets from a nano-MgO template and $\mathrm{ZnCl}_{2}$ activation: morphology, porosity and supercapacitance performance. RSC Adv. 6, 71360-71369 (2016).

20. Zhu, S. et al. Soluble salt self-assembly-assisted synthesis of three-dimensional hierarchical porous carbon networks for supercapacitors. J. Mater. Chem. A 3, 22266-22273 (2015).

21. Zhu, S. et al. Synthesis of 2D/3D carbon hybrids by heterogeneous space-confined effect for electrochemical energy storage. J. Mater. Chem. A 5, 19175-19183 (2017).

22. Wei, X., Wan, S. \& Gao, S. Self-assembly-template engineering nitrogen-doped carbon aerogels for high-rate supercapacitors. Nano Energy 28, 206-215 (2016).

23. Wei, X., Zou, H. \& Gao, S. Chemical crosslinking engineered nitrogen-doped carbon aerogels from polyaniline-boric acid-polyvinyl alcohol gels for high performance electrochemical capacitors. Carbon 123, 471-480 (2017).

24. Zhou, Y. et al. Multi-functional graphene/carbon nanotube aerogels for its applications in supercapacitor and direct methanol fuel cell. Electrochim. Acta. 264, 12-19 (2018).

25. Biener, J. et al. Advanced carbon aerogels for energy applications. Energy Environ. Sci. 4, 656-667 (2011).

26. Ramakrishnan, P. \& Shanmugam, S. Nitrogen-doped porous multi-nano-channel nanocarbons for use in high-performance supercapacitor applications. ACS Sustainable Chem. Eng. 4, 2439-2448 (2016).

27. Zou, J. et al. Ultralight multiwalled carbon nanotube aerogel. ACS Nano. 4, 7293-7302 (2010).

28. Tang, Y., Liu, L., Wang, X., Zhou, H. \& Jia, D. High-yield bamboo-like porous carbon nanotubes with high-rate capability as anodes for lithium-ion batteries. RSC Adv. 4, 4852-4857 (2014).

29. Hu, H., Zhao, Z., Wan, W., Gogotsi, Y. \& Qiu, J. Ultralight and highly compressible graphene aerogels. Adv. Mater. 25, 2219-2223 (2013).

30. Li, J., Li, X., Xiong, D., Wang, L. \& Li, D. Enhanced capacitance of boron-doped graphene aerogels for aqueous symmetric supercapacitors. Appl. Surf. Sci. 475, 285-293 (2019).

31. Xu, X. et al. Flexible, highly graphitized carbon aerogels based on bacterial cellulose/lignin: catalyst-free synthesis and its application in energy storage devices. Adv. Funct. Mater. 25, 3193-3202 (2015).

32. Fellinger, T. P., White, R. J., Titirici, M. M. \& Antonietti, M. Borax-mediated formation of carbon aerogels from glucose. Adv. Funct. Mater. 22, 3254-3260 (2012).

33. Guo, M. et al. Hierarchical porous carbon spheres constructed from coal as electrode materials for high performance supercapacitors. RSC Adv. 7, 45363-45368 (2017).

34. Song, X. et al. Free standing needle-like polyaniline-coal based carbon nanofibers composites for flexible supercapacitor. Electrochim. Acta. 206, 337-345 (2016). 
35. Li, J., Cao, Y., Wang, L. \& Jia, D. Cost-effective synthesis of bamboo-structure carbon nanotubes from coal for reversible lithium storage. RSC Adv. 7, 34770-34775 (2017).

36. Zhang, S. et al. Construction of hierarchical porous carbon nanosheets from template-assisted assembly of coal-based graphene quantum dots for high performance supercapacitor electrodes. Mater. Today Energy. 6, 36-45 (2017).

37. Gao, S. et al. Coal-based hierarchical porous carbon synthesized with a soluble salt self-assembly-assisted method for high performance supercapacitors and Li-ion batteries. ACS Sustainable Chem. Eng. 6, 3255-3263 (2018).

38. Guo, M. et al. Coal derived porous carbon fibers with tunable internal channels for flexible electrodes and organic matter absorption. J. Mater. Chem. A 3, 21178-21184 (2015).

39. Wen, Z. et al. Crumpled nitrogen-doped graphene nanosheets with ultrahigh pore volume for high-performance supercapacitor. Adv. Mater. 24, 5610-5616 (2012).

40. Wang, H., Gao, Q. \& Hu, J. High hydrogen storage capacity of porous carbons prepared by using activated carbon. J. Am. Chem. Soc. 131, 7016-7022 (2009).

41. Liu, X. \& Antonietti, M. Moderating black powder chemistry for the synthesis of doped and highly porous graphene nanoplatelets and their use in electrocatalysis. Adv. Mater. 25, 6284-6290 (2013).

42. Zhao, G. et al. One-step production of O-N-S co-doped three-dimensional hierarchical porous carbons for high-performance supercapacitors. Nano Energy. 47, 547-555 (2018).

43. Yu, Q. et al. Control and characterization of individual grains and grain boundaries in graphene grown by chemical vapour deposition. Nat. Mater. 10, 443-449 (2011).

44. Shimodaira, N. \& Masui, A. Raman spectroscopic investigations of activated carbon materials. J. Appl. Phys. 92, 902-909 (2002).

45. Pan, H., Yin, X., Xue, J., Cheng, L. \& Zhang, L. In-situ synthesis of hierarchically porous and polycrystalline carbon nanowires with excellent microwave absorption performance. Carbon 107, 36-45 (2016).

46. Chaunchaiyakul, S. et al. Nanoscale analysis of multiwalled carbon nanotube by tip-enhanced raman spectroscopy. Carbon 99, 642-648 (2016).

47. Zhang, Z., Zhou, Z., Peng, H., Qin, Y. \& Li, G. Nitrogen- and oxygen-containing hierarchical porous carbon frameworks for highperformance supercapacitors. Electrochim. Acta. 134, 471-477 (2014).

48. Arrigo, R. et al. Tuning the acid/base properties of nanocarbons by functionalization via amination. J. Am. Chem. Soc. 132, 9616-9630 (2010).

49. Chen, Z. et al. Self-Biotemplate preparation of hierarchical porous carbon with rational mesopore ratio and high oxygen content for an ultrahigh energy-density supercapacitor. ACS Sustainable Chem. Eng. 6, 7138-7150 (2018).

50. Sevilla, M. \& Mokaya, R. Energy storage applications of activated carbons: supercapacitors and hydrogen storage. Energy Environ. Sci. 7, 1250-1280 (2014).

51. Shao, J. et al. In-situ $\mathrm{MgO}\left(\mathrm{CaCO}_{3}\right)$ templating coupled with $\mathrm{KOH}$ activation strategy for high yield preparation of various porous carbons as supercapacitor electrode materials. Chem. Eng. J. 321, 301-313 (2017).

52. Zhu, S. et al. Soluble salt self-assembly-assisted synthesis of three-dimensional hierarchical porous carbon networks for supercapacitors. J. Mater. Chem. A 3, 22266-22273 (2015).

53. Wang, H., Yu, S. \& Xu, B. Hierarchical porous carbon materials prepared using nano- $\mathrm{ZnO}$ as a template and activation agent for ultrahigh power supercapacitors. Chem. Commun. 52, 11512-11515 (2016).

54. Raj, C. et al. High electrochemical capacitor performance of oxygen and nitrogen enriched activated carbon derived from the pyrolysis and activation of squid gladius chitin. J. Power Sources 386, 66-76 (2018).

55. Chmiola, J. et al. Anomalous increase in carbon capacitance at pore sizes less than 1 nanometer. Science. 313, 1760-1763 (2006)

56. Peng, H. et al. Nitrogen-doped interconnected carbon nanosheets from pomelo mesocarps for high performance supercapacitors. Electrochim. Acta. 190, 862-871 (2016).

57. Liu, R. et al. An interface-induced co-assembly approach towards ordered mesoporous carbon/graphene aerogel for highperformance supercapacitors. Adv. Funct. Mater. 25, 526-533 (2015).

58. Song, W., Song, K. \& Fan, L. A versatile strategy toward binary three-dimensional architectures based on engineering graphene aerogels with porous carbon fabrics for supercapacitors. ACS Appl. Mater. Interf. 7, 4257-4264 (2015).

59. Shao, J. et al. Facile preparation of 3D nanostructured $\mathrm{O} / \mathrm{N}$ co-doped porous carbon Constructed by internected carbon nanosheets for excellent-performance supercapacitors. Electrochim. Acta. 222, 793-805 (2016).

60. He, Y., Wang, L. \& Jia, D. Coal/PAN interconnected carbon nanofibers with excellent energy storage performance and electrical conductivity. Electrochim. Acta. 194, 239-245 (2016).

61. Huang, J., Sumpter, B. \& Meunier, V. Theoretical model for nanoporous carbon supercapacitors. Angew. Chem. Int. Ed. 47, 520-524 (2008).

\section{Acknowledgements}

This work is supported by the National Natural Science Foundation of China (201972123, U1703251, and 21861037), Program for Tianshan Innocative Research Team of Xinjiang Uygur Autonomous Region (2018D14002), the Open Fund of the Key Laboratory of Xinjiang Uygur Autonomous Region (2017D040414), Scientific Research Program of the Higher Education Institution of Xinjiang (XJEDU2017A001).

\section{Author contributions}

Yan Lv performed the experiments, discussed the results and wrote the manuscript. Xueyan Wu, Jixi Guo and Dianzeng Jia supervised the work, discussed the results and co-wrote the manuscript. Lili Ding and Shengchao Hou helped for the sample measurements. Nannan Guo contributed to the revision and language polishing of the article after peer review. Fenglian Tong and Hongbo Zhang contributed to the scientifc discussion and manuscript revisions.

\section{Competing interests}

The authors declare no competing interests.

\section{Additional information}

Supplementary information is available for this paper at https://doi.org/10.1038/s41598-020-64020-5.

Correspondence and requests for materials should be addressed to J.G. or D.J.

Reprints and permissions information is available at www.nature.com/reprints.

Publisher's note Springer Nature remains neutral with regard to jurisdictional claims in published maps and institutional affiliations. 
(c) (i) Open Access This article is licensed under a Creative Commons Attribution 4.0 International License, which permits use, sharing, adaptation, distribution and reproduction in any medium or format, as long as you give appropriate credit to the original author(s) and the source, provide a link to the Creative Commons license, and indicate if changes were made. The images or other third party material in this article are included in the article's Creative Commons license, unless indicated otherwise in a credit line to the material. If material is not included in the article's Creative Commons license and your intended use is not permitted by statutory regulation or exceeds the permitted use, you will need to obtain permission directly from the copyright holder. To view a copy of this license, visit http://creativecommons.org/licenses/by/4.0/.

(C) The Author(s) 2020 